\title{
The Effects of Sociodemographic Factors on Psychiatric Diagnosis
}

\author{
Mal Rye Choi ${ }^{1,2}$, Hun-Jeong Eun', Tai P. Yoo², Youngmi Yun ${ }^{3}$, \\ Christopher Wood ${ }^{2}$, Michael Kase ${ }^{2}$, Jong-II Park ${ }^{4}$ and Jong-Chul Yang $4,5 \bowtie$ \\ ${ }^{1}$ Department of Neuropsychiatry, Presbyterian Medical Center, Jeonju, Republic of Korea \\ ${ }^{2}$ Department of Psychiatry, Kern Medical Center, Bakersfield, CA, USA \\ ${ }^{3}$ California State University at Bakersfield, Bakersfield, CA, USA \\ ${ }^{4}$ Department of Psychiatry, Chonbuk National University Hospital and Research Institute of Clinical Medicine, Jeonju, Republic of Korea \\ ${ }^{5}$ Department of Psychiatry, Chonbuk National University Medical School and Institute for Medical Sciences, Jeonju, Republic of Korea
}

Objective Several studies have reported that ethnic differences influence psychiatric diagnoses. Some previous studies reported that African Americans and Hispanics are diagnosed with schizophrenia spectrum disorders more frequently than Caucasians, and that Caucasians are more likely to be diagnosed with affective disorders than other ethnic groups. We sought to identify associations between sociodemographic factors and psychiatric diagnosis.

Methods We retrospectively examined the medical records of all psychiatric inpatients (ages over 18 years) treated at Kern county mental hospital ( $n=2,051)$ between July 2003 and March 2007 for demographic, clinical information, and discharge diagnoses.

Results African American and Hispanic males were more frequently diagnosed with schizophrenia spectrum disorders than Caucasians, whereas Caucasian females were more frequently diagnosed with affective disorders than females in the other ethnic groups, suggesting that patient ethnicity and gender may influence clinical diagnoses. Demographic variables, that is, a lower education, failure of marriage, homelessness, and low quality insurance, were found to be significantly associated with a diagnosis of schizophrenia spectrum disorders after adjusting for clinical variables. And, the presence of a family psychiatric history, failure of marriage, not-homelessness, and quality insurance were found to be associated with a diagnosis of affective disorders.

Conclusion Our results show that these demographic factors, including ethnicity, have effects on diagnoses in psychiatric inpatients. Furthermore, these variables may help prediction of psychiatric diagnoses.

Psychiatry Investig 2012;9:199-208

Key Words Ethnicity, Demographic variables, Clinical variables, Psychiatric diagnoses, Schizophrenia, Affective disorders.

\section{INTRODUCTION}

A number of previous studies have reported that ethnicity influences psychiatric diagnosis. In several studies, African American patients have been found to be diagnosed with schizophrenia spectrum disorders more frequently and depression less frequently during routine clinical assessments compared with similar Caucasian patients. ${ }^{1-12}$ Likewise, Hispanic bipolar patients might also be at greater risk than Caucasians for being misdiagnosed as having schizophrenia. ${ }^{13}$ Further-

Received: February 11, 2012 Revised: April 27, 2012

Accepted: April 27, 2012 Available online: September 6, 2012

$\bowtie$ Correspondence: Jong-Chul Yang, MD, PhD

Department of Psychiatry, Chonbuk National University Medical School, 567 Baekje-daero, Deokjin-gu, Jeonju 561-756, Republic of Korea

Tel: +82-63-250-1390, Fax: +82-63-275-3157, E-mail: yangjc@jbnu.ac.kr

(c) This is an Open Access article distributed under the terms of the Creative Commons Attribution Non-Commercial License (http://creativecommons.org/licenses/bync/3.0) which permits unrestricted non-commercial use, distribution, and reproduction in any medium, provided the original work is properly cited. more, this apparent misdiagnosis of schizophrenia may lead to inadequate recognition and treatment of mood disorders, ${ }^{5}$ which, in turn results in the lower use of mental health treatment for mood disorders among Hispanics and African Americans than among Caucasians. ${ }^{14}$ In addition, when strict diagnostic criteria were applied, fewer differences were found between African American and Caucasian patients in terms of rates of psychotic disorders, ${ }^{13,15,16}$ and an Epidemiologic Catchment Area study showed equal distributions for the prevalences of major psychotic disorders among ethnic groups after controlling for sociodemographic variables. ${ }^{2,17}$ Accordingly, the overdiagnosis of schizophrenia is generally believed to be the result of misdiagnosis rather than any ethnic difference in prevalence. ${ }^{5}$

However, the reasons why clinicians overdiagnose schizophrenia in African American and Hispanics are unclear. Some have hypothesized that social and cultural differences between African American and Caucasian clinicians are responsible 
for this discrepancy, ${ }^{8,19,20}$ whereas others have hypothesized that it is due to ethnic differences in the symptomatic presentations of affective and psychotic disorders. ${ }^{26}$ Previous studies have shown that African Americans reported higher frequencies and severities of hallucinations and paranoid symptoms than Caucasians. ${ }^{9,11,15,18,21}$ Likewise, Mexican-American schizophrenia patients were found to be more likely to report physical symptoms than Caucasian patients. ${ }^{22}$ In contrast, Caucasian patients reported experiencing nervous tension, a greater frequency of persecutory delusions, and a blunted affect. ${ }^{22} \mathrm{Th}$ ese differences between ethnic groups in terms of the symptomatic presentations of affective and psychotic disorders could explain why schizophrenia is over-diagnosed in African-American and Hispanics.

Furthermore, research and clinical diagnoses of schizophrenia are less likely to agree in African Americans than in Caucasians, ${ }^{9}$ and affective symptoms identified during structured interviews are less commonly recorded by clinicians for African American than Caucasian patients. These findings suggest that clinicians often fail to identify affective symptoms in African American patients with psychosis. Furthermore, African American patients with affective disorder in the USA have been misdiagnosed as having schizophrenia, because Schneiderian first rank or other psychotic symptoms may be common to such patients. ${ }^{6-8,12,13,20}$ This risk of misdiagnosis appears to be greater in African American men than women. ${ }^{10,12,20}$ In addition, African-Caribbean's and Africans were found to be significantly less likely to have experienced a depressive episode before onset of first mania, but to have experienced more severe psychotic symptoms at first mania, although no differences in mood incongruence or first rank symptoms were observed between the two groups. ${ }^{23}$

Researchers have also reported that African Americans are more frequently diagnosed with disorders in the schizophrenia spectrum than Caucasians or Hispanics. Furthermore, Hispanics have been reported to be disproportionately diagnosed to have major depression, ${ }^{24}$ which suggests that an examination of Hispanic psychiatric patients might result in a similar pattern of schizophrenia misdiagnosis.

Most studies have used ethnic comparisons between African Americans and Caucasians. In contrast, the present study was designed to simultaneously compare relationships between ethnicity and the frequencies of major psychiatric diagnoses in a sample of African American, Hispanic, and Caucasian psychiatric inpatients. We considered that use of different ethnicities and methods of acquiring diagnostic data would help us answer the following questions:

1) Are previous findings of higher rates of schizophrenia spectrum disorders among African American patients replicated? Do Hispanic patients have a psychiatric diagnosis pat- tern similar to that of African Americans?

2) Among the selected demographic and clinical variables, which are significantly associated with psychiatric diagnosis? Do demographic variables significantly enhance the likelihood of a psychiatric diagnosis based on predictions using clinical variables?

\section{METHODS}

Kern County is located in southern-central California, occupying 8,173 square miles. It is the third largest in-land area in the United State. It is the eighth fastest growing business market in the United States. It has an ethnically diverse population of nearly 780,117 people. The ethnic groups are: African American (5.1\% of adult population), Caucasians (63.7\% of adult population), and Hispanic/Latino (44.4\% of adult population). ${ }^{25}$

We retrospectively reviewed demographic and clinical information of the Diagnostic and Statistical Manual of Mental Disorder, Fourth Edition (American Psychiatric Association) Axis I and Axis II discharge diagnoses from the discharge summaries of all patients admitted to the county mental hospital between July 2003 and March 2007. Ethnic comparisons were limited to African Americans, Caucasians, and Hispanics, as other ethnic groups were not adequately represented.

The data for this study were obtained from quality assurance and medical records databases in the hospital management information system. All of these records were abstracted from the patient's hospital medical records and social workers reports. Diagnoses were obtained from discharge summaries. We included adults psychiatric inpatients with diagnosis of major depressive disorder, bipolar disorder, schizoaffective disorder, schizophreniform disorder, schizophrenia, psychotic disorder not otherwise specified, panic disorder, social phobia, post traumatic stress disorder, generalized anxiety disorder, specific phobia, substance abuse and dependence, alcohol abuse and dependence, and personality disorder, who received treatment at least once between July 2003 and March 2007. Diagnoses that were listed as "rule out" diagnoses and another psychiatric disorder were not included in this analysis. To reduce the number of variables in some analyses, diagnoses are combined into specific categories. Major depressive disorder and bipolar disorder were categorized as "affective disorder;" Schizophrenia, schizophreniform disorder, schizoaffective disorder, and psychotic disorder not otherwise specified were categorized as "schizophrenia spectrum disorder;" Alcohol abuse and dependence, and substance abuse and dependence were categorized as "alcohol and substance use disorder;" Panic disorder, social phobia, post traumatic stress disorder, generalized anxiety disorder, specific phobia were categorized as "anxiety disorder," retrospectively. The Institu- 
tional Review Board of the Kern Medical Center gave permission for this study.

Data analyses were performed using SPSS, Version. 14.0. Ethnic differences in eight demographic and eight clinical variables were explored separately using chi-square analyses (a significance level of 0.05 was predetermined). This was followed by logistic regression analyses of all the variables in order to examine factors associated with psychiatric diagnosis. A series of binary logistic analyses was conducted through SPSS Binary Logistic on major psychiatric diagnoses as dependent variables: schizophrenia (1) versus other (0); and affective disorder (1) versus other (0), and all clinical and demographic variables as predictor variables.

\section{RESULTS}

\section{Descriptive and chi-square results}

Demographic and clinical characteristics by ethnicity and gender of the subjects are presented in Table 1 and Table 2, respectively. Eight demographic variables (age, employment, education, marital status, living situation, insurance, residence, and religion) and eight clinical variables (final diagnosis, type of admission, discharge GAF, past psychiatric history, family psychiatric history, aggression type, clinician race, and length of hospitalization) were used in this study. We analyzed 2,051 patients and the ethnic composition of subjects was 58.4\% Caucasian, 29.6\% Hispanic, and 12\% African American. The subjects were evenly consisted of males and females (50.7\% for males and $49.3 \%$ for females). Majority of the subjects were unemployed, never married, involuntarily admitted, urban residence, not-homelessness, medically insured, and below or at most high school graduates. As for the diagnostic distribution, $86 \%$ of the subjects $(\mathrm{n}=1,760)$ received a schizophrenia spectrum disorder or an affective disorder.

Chi-square results in Table 1 and Table 2 showed differential distributional patterns among the three racial groups in some variables: age, education, marital status, living situation, insurance, residence, family psychiatric history. Specifically, Hispanics were younger $\left(\chi^{2}=75.5, \mathrm{df}=4, \mathrm{p}<0.001\right.$; in addition, it was confirmed by a significant ANOVA result of $\mathrm{F}=75.47$, $\mathrm{df}=2, \mathrm{p}<0.001)$ and less educated $\left(\mathrm{X}^{2}=48.31, \mathrm{df}=6, \mathrm{p}<0.001\right)$ compared with Caucasians. Caucasians, especially females, tended to have more family psychiatric history $\left(X^{2}=40.52, \mathrm{df}=2\right.$, $\mathrm{p}<0.001)$.

Chi-square analyses (a $5 \times 3$ contingency table) in Table 2 indicates a significant ethnic difference on the five diagnoses distributions $\left(X^{2}=93.13, \mathrm{df}=8, \mathrm{p}<0.001\right)$. To identify specific ethnic differences in diagnosis, analysis (a $2 \times 3$ contingency table) on each diagnosis was followed. There were differential diagnostic patterns for schizophrenia spectrum disorder $\left(\chi^{2}=\right.$
81.36, $\mathrm{df}=2, \mathrm{p}<0.001)$ and affective disorder $\left(\chi^{2}=83.79, \mathrm{df}=2\right.$, $\mathrm{p}<0.001)$ among the ethnic groups. Specifically, Hispanics and African Americans displayed a higher rate of schizophrenia spectrum disorder and a lower rate of affective disorder compared with Caucasians, who had a higher rate of affective disorder. Yet, there were no ethnic differences in other diagnoses.

As for the ethnicity of the clinician, Asian clinicians tended to diagnose with schizophrenia spectrum disorders more than affective disorders, while Caucasian clinicians tended to diagnose with affective disorders more than schizophrenia spectrum disorders $\left(\chi^{2}=36.561, \mathrm{df}=12, \mathrm{p}<0.001\right)$. As for past psychiatric history, Hispanic and African American patients had a higher rate of schizophrenia spectrum disorder and a lower rate of affective disorder compared with Caucasian patients, who had a higher rate of affective disorder $\left(\chi^{2}=27.084, \mathrm{df}=10\right.$, $\mathrm{p}<0.003)$.

For a closer investigation of a gender effect on the ethnic differences in the major diagnoses (i.e., schizophrenia spectrum disorder and affective disorder), a further analysis on the two diagnoses was conducted. The results (Table 3 ) showed that all of the largest residuals were in the Caucasian female, Hispanic male and African American male columns. Caucasian females had much more affective disorder and fewer schizophrenia spectrum disorder, while Hispanic and African American males had far more schizophrenia spectrum disorder and fewer affective disorder than would be expected if there were no ethnic differences on the diagnosis. However, this association between ethnicity and psychiatric diagnosis does not indicate that being African American or Hispanic male causes more schizophrenia spectrum disorder.

In order to examine the possible effect of socioeconomic factor on the ethnic differences in the diagnoses, only private insurance holders were selected and analyzed (although socioeconomic status was not specifically recorded, this was suggested by insurance status). Unlike previous studies, ethnic differences still existed $\left(\chi^{2}=98.66, d f=5, p<0.001\right)$ even after controlling for the socioeconomic level, which showed similar diagnostic pattern to the uncontrolled group. Within the group of subjects with private insurance, there were more African Americans and Hispanics diagnosed with schizophrenia spectrum disorders than Caucasians.

\section{Logistic regression results}

Within each category of schizophrenia spectrum disorder and affective disorder, we explored factors associated with the diagnosis and examined their odds ratios. Eight variables (family psychiatric history, length of hospitalization, gender, education, marital status, ethnicity, living situation, and insurance) were significantly associated with the diagnosis of schizophrenia spectrum disorder $\left(\chi^{2}=291.29, \mathrm{df}=15, \mathrm{p}<0.001\right)$ 
Table 1. Demographic characteristics and chi-square analyses of ethnic differences

\begin{tabular}{|c|c|c|c|c|c|c|c|c|}
\hline \multirow[b]{2}{*}{ Variables } & \multicolumn{2}{|c|}{ Caucasians } & \multicolumn{2}{|c|}{ Hispanics } & \multicolumn{2}{|c|}{ African Americans } & \multirow[b]{2}{*}{$\chi^{2}(\mathrm{df})$} & \multirow[b]{2}{*}{$\mathrm{p}$-value } \\
\hline & $\begin{array}{c}\text { Males } \\
(\mathrm{N}=609)\end{array}$ & $\begin{array}{l}\text { Females } \\
(\mathrm{N}=589)\end{array}$ & $\begin{array}{c}\text { Males } \\
(\mathrm{N}=299)\end{array}$ & $\begin{array}{l}\text { Females } \\
(\mathrm{N}=308)\end{array}$ & $\begin{array}{c}\text { Males } \\
(\mathrm{N}=123)\end{array}$ & $\begin{array}{l}\text { Females } \\
(\mathrm{N}=123)\end{array}$ & & \\
\hline Age (yr) & & & & & & & $75.5(4)$ & $<0.001^{*}$ \\
\hline $18-34$ & $216(35.5)$ & $148(25.1)$ & $117(59.2)$ & $131(42.5)$ & $45(36.6)$ & $35(28.5)$ & & \\
\hline $35-64$ & $381(62.5)$ & $408(69.3)$ & $119(39.8)$ & $168(54.5)$ & $77(62.6)$ & $83(67.5)$ & & \\
\hline$\geq 65$ & $12(2.0)$ & $33(5.6)$ & $3(1.0)$ & $9(2.9)$ & $1(0.8)$ & $5(4.1)$ & & \\
\hline Employment & & & & & & & $3.15(4)$ & 0.532 \\
\hline Unemployed & $542(89.0)$ & $533(90.5)$ & $266(89.0)$ & $269(87.3)$ & $113(91.9)$ & $113(91.9)$ & & \\
\hline Part-time & $14(2.3)$ & $6(1.0)$ & $2(0.6)$ & $12(3.9)$ & $3(2.4)$ & & & \\
\hline Full-time & $53(8.7)$ & $50(8.5)$ & $31(10.4)$ & $27(8.8)$ & $7(5.7)$ & $10(8.1)$ & & \\
\hline Education & & & & & & & $48.31(6)$ & $<0.001^{*}$ \\
\hline$<$ High school & $215(35.3)$ & $208(35.3)$ & $149(49.8)$ & $127(41.2)$ & $51(41.5)$ & $45(36.6)$ & & \\
\hline High school & $166(27.3)$ & $155(26.3)$ & $85(28.4)$ & $80(26.0)$ & $30(24.4)$ & $36(29.3)$ & & \\
\hline 2 yr college & $77(12.6)$ & $93(15.8)$ & $13(4.3)$ & $26(8.4)$ & $8(6.5)$ & $21(17.1)$ & & \\
\hline$>4$ yr college & $66(10.8)$ & $52(8.8)$ & $14(4.7)$ & $13(4.2)$ & $7(5.7)$ & $7(5.7)$ & & \\
\hline Missing data & $85(14.0)$ & $81(13.8)$ & $38(12.7)$ & $62(20.1)$ & $27(22.0)$ & $14(11.4)$ & & \\
\hline Marital status & & & & & & & $48.7(4)$ & $<0.001^{*}$ \\
\hline Never married & $434(71.3)$ & $207(35.1)$ & $234(78.3)$ & $160(51.9)$ & $92(74.8)$ & $76(61.8)$ & & \\
\hline Divorced, separated & $108(17.7)$ & $257(43.6)$ & $30(10.0)$ & $76(24.7)$ & $25(20.3)$ & $28(22.8)$ & & \\
\hline Married & $58(9.5)$ & $110(18.7)$ & $30(10.0)$ & $67(21.8)$ & $4(3.3)$ & $17(13.8)$ & & \\
\hline Missing data & $9(1.5)$ & $15(2.5)$ & $5(1.7)$ & $5(1.6)$ & $2(1.6)$ & $2(1.6)$ & & \\
\hline Living situation & & & & & & & $14.57(2)$ & $<0.001^{*}$ \\
\hline Homeless & $239(39.3)$ & $178(30.3)$ & $85(28.4)$ & $80(26.0)$ & $61(49.6)$ & $35(27.5)$ & & \\
\hline Not homelessness & $370(60.7)$ & $411(69.7)$ & $214(71.6)$ & $228(74.0)$ & $62(50.4)$ & $88(72.5)$ & & \\
\hline Insurance & & & & & & & $37.92(4)$ & $<0.001^{*}$ \\
\hline Medical health & $207(34.0)$ & $254(43.1)$ & $125(42.0)$ & $164(53.2)$ & $64(52.0)$ & $69(56.1)$ & & \\
\hline Medicare, HMO & $206(33.8)$ & $187(31.7)$ & $69(22.8)$ & $67(21.5)$ & $27(22.0)$ & $31(25.2)$ & & \\
\hline Private insurance & $196(32.2)$ & $148(25.2)$ & $105(35.2)$ & $78(25.3)$ & $32(26.0)$ & $23(18.7)$ & & \\
\hline Residence & & & & & & & $33.07(2)$ & $<0.001^{*}$ \\
\hline Rural & $232(38.1)$ & $218(37.0)$ & $129(43.1)$ & $132(42.9)$ & $22(17.9)$ & $30(24.4)$ & & \\
\hline Urban & $349(57.3)$ & $362(61.5)$ & $165(55.2)$ & $176(57.1)$ & $96(78.0)$ & $88(71.5)$ & & \\
\hline Missing data & $28(4.6)$ & $9(1.5)$ & $5(1.7)$ & & $5(4.1)$ & $5(4.1)$ & & \\
\hline Religion & & & & & & & $10.81(2)$ & 0.004 \\
\hline None & $354(58.1)$ & $297(50.4)$ & $163(54.5)$ & $143(46.4)$ & $62(50.4)$ & $50(40.7)$ & & \\
\hline Any religion & $219(36.0)$ & $254(43.1)$ & $123(41.1)$ & $157(51.0)$ & $54(43.9)$ & $69(56.1)$ & & \\
\hline Missing data & $36(5.9)$ & $38(6.5)$ & $13(4.3)$ & $8(2.6)$ & $7(5.7)$ & $4(3.3)$ & & \\
\hline
\end{tabular}

Data are given as number (percentage) of subjects. Percentages may not total 100 because of rounding. Missing data were not counted for chisquare analyses. Significant results are marked by an asterisk $(*)$. HMO: Health Maintenance Organization

according to Wald criterion using a criterion $\alpha=0.003$ to compensate for inflated Type I error rate with 15 predictors (Table 4). About $70 \%$ of subjects were correctly classified by all the predictors. Schizophrenia spectrum disorders generally decreased with educational level (OR=0.799; 95\% CI, 0.7070.903 ), marriage ( $\mathrm{OR}=0.672 ; 95 \% \mathrm{CI}, 0.563-0.802)$, not homelessness (i.e., home-staying; $\mathrm{OR}=0.66$; 95\% CI, 0.514$0.847)$, and quality insurance ( $\mathrm{OR}=0.743$; $95 \% \mathrm{CI}, 0.645-0.855)$.
For gender, males were $40 \%$ more likely to be diagnosed as having schizophrenia spectrum disorder than females (OR= 0.609; 95\% CI, 0.48-0.773). When comparing Hispanics and Caucasians, Hispanics was about twice more likely to be diagnosed as having schizophrenia spectrum disorder than Caucasians (OR=2.06; 95\% CI, 1.87-2.24). An odds ratio comparing African Americans with Caucasians was 2.79 (95\% CI, 2.52-3.05), which indicated African Americans were three 
MR Choi et al.

Table 2. Clinical characteristics and chi-square analyses of ethnic differences

\begin{tabular}{|c|c|c|c|c|c|c|c|c|}
\hline \multirow[b]{2}{*}{ Variables } & \multicolumn{2}{|c|}{ Caucasians } & \multicolumn{2}{|c|}{ Hispanics } & \multicolumn{2}{|c|}{ African Americans } & \multirow[b]{2}{*}{$\chi^{2}(\mathrm{df})$} & \multirow[b]{2}{*}{ p-value } \\
\hline & $\begin{array}{c}\text { Males } \\
(\mathrm{N}=609)\end{array}$ & $\begin{array}{l}\text { Females } \\
(\mathrm{N}=589)\end{array}$ & $\begin{array}{c}\text { Males } \\
(\mathrm{N}=299)\end{array}$ & $\begin{array}{l}\text { Females } \\
(\mathrm{N}=308)\end{array}$ & $\begin{array}{c}\text { Males } \\
(\mathrm{N}=123)\end{array}$ & $\begin{array}{l}\text { Females } \\
(\mathrm{N}=123)\end{array}$ & & \\
\hline Diagnoses & & & & & & & $93.13(8)$ & $<0.001^{*}$ \\
\hline Schizophrenia spectrum $\mathrm{d} / \mathrm{o}$ & $252(41.4)$ & $198(33.6)$ & $199(66.6)$ & $136(44.1)$ & $87(70.5)$ & $68(55.4)$ & $81.36(2)$ & $<0.001^{*}$ \\
\hline Affective disorder & $275(45.2)$ & $299(50.9)$ & $68(22.6)$ & $125(40.5)$ & $14(11.5)$ & $39(31.4)$ & $83.79(2)$ & $<0.001^{*}$ \\
\hline Alcohol/Substance use d/o & $21(3.5)$ & $11(1.9)$ & $8(2.7)$ & $7(2.3)$ & $4(3.3)$ & $2(1.7)$ & $0.09(2)$ & 0.957 \\
\hline Anxiety disorder & $10(1.7)$ & $21(3.4)$ & $5(1.7)$ & $10(3.3)$ & $3(2.5)$ & $2(1.7)$ & $0.21(2)$ & 0.907 \\
\hline Personality disorder & $51(8.3)$ & $61(10.2)$ & $19(6.4)$ & $30(9.9)$ & $15(12.3)$ & $12(9.9)$ & $1.82(2)$ & 0.403 \\
\hline Type of admission & & & & & & & $0.88(2)$ & 0.644 \\
\hline Involuntary & $554(91.0)$ & $559(94.9)$ & $276(92.3)$ & $293(95.1)$ & $114(92.7)$ & $118(95.9)$ & & \\
\hline Voluntary & $55(9.0)$ & $30(5.1)$ & $23(7.7)$ & $15(4.9)$ & $9(7.3)$ & $5(4.1)$ & & \\
\hline Discharge GAF & & & & & & & $10.04(8)$ & 0.262 \\
\hline Inability of functioning & $67(10.9)$ & $52(8.7)$ & $32(10.5)$ & $27(8.8)$ & $15(12.4)$ & $13(10.7)$ & & \\
\hline Serious impairment & $139(22.9)$ & $171(29.1)$ & $75(25.0)$ & $80(26.1)$ & $32(25.6)$ & $35(28.7)$ & & \\
\hline Major impairment & $286(47.0)$ & $261(44.4)$ & $143(48.0)$ & $145(47.2)$ & $53(43.0)$ & $45(36.1)$ & & \\
\hline Moderate difficulty & $93(15.2)$ & $87(14.7)$ & $40(13.5)$ & $45(14.7)$ & $22(18.2)$ & $27(22.1)$ & & \\
\hline Mild impairment & $24(4.0)$ & $18(3.1)$ & $9(3.0)$ & $10(3.3)$ & $1(0.8)$ & $3(2.5)$ & & \\
\hline Family psychiatric history & & & & & & & $40.52(2)$ & $<0.001^{*}$ \\
\hline Parent, sibling, children & $223(36.6)$ & $279(47.4)$ & $74(24.7)$ & $89(28.9)$ & $37(30.1)$ & $45(36.6)$ & & \\
\hline None & $386(63.4)$ & $310(52.6)$ & $225(71.7)$ & $219(71.7)$ & $86(69.9)$ & $78(63.4)$ & & \\
\hline Aggression type & & & & & & & $21.46(6)$ & 0.002 \\
\hline DTS and DTO & $28(4.6)$ & $26(4.4)$ & $24(8.0)$ & $24(7.8)$ & $3(2.4)$ & $5(4.1)$ & & \\
\hline DTO & $48(7.9)$ & $37(6.3)$ & $35(11.7)$ & $20(6.5)$ & $16(13.0)$ & $17(13.8)$ & & \\
\hline DTS & $113(18.6)$ & $118(20.0)$ & $52(17.4)$ & $46(14.9)$ & $22(17.9)$ & $23(18.7)$ & & \\
\hline None & $73(12.0)$ & $87(14.8)$ & $46(15.4)$ & 45 (14.6) & $16(13.0)$ & $18(14.6)$ & & \\
\hline Missing data & $347(57.0)$ & $321(54.5)$ & $142(47.5)$ & $173(56.2)$ & $66(53.7)$ & $60(48.8)$ & & \\
\hline Physician race & & & & & & & $3.39(6)$ & 0.758 \\
\hline Caucasians & $65(10.6)$ & $41(6.8)$ & $18(6.1)$ & $31(10.2)$ & $14(11.4)$ & $14(11.5)$ & & \\
\hline Hispanics & $52(8.4)$ & $57(9.7)$ & $21(7.1)$ & $28(9.2)$ & $9(7.3)$ & $11(9.0)$ & & \\
\hline Asians & 255 (41.9) & $228(38.8)$ & $146(48.8)$ & $107(34.8)$ & $64(52.0)$ & $39(31.1)$ & & \\
\hline Other & $237(39.1)$ & $263(44.7)$ & $114(38.0)$ & $142(45.9)$ & $36(29.3)$ & $59(48.4)$ & & \\
\hline LOH (days) & & & & & & & ANOI & A test \\
\hline Mean (Std. Dev) & $7.18(5.4)$ & $7.07(5.4)$ & $7.82(5.2)$ & $8.1(6.04)$ & $7.29(5.4)$ & $6.89(4.9)$ & $\begin{array}{r}\mathrm{F}=2.13 \\
\mathrm{p}=0\end{array}$ & $\begin{array}{l}\mathrm{df}=5, \\
06\end{array}$ \\
\hline
\end{tabular}

Data are given as number (percentage) of subjects. Percentages may not total 100 because of rounding. Missing data were not counted for chisquare analyses. Significant results are marked as an asterisk $\left(^{*}\right)$. LOH: length of hospitalization, DTO: dangerous to others, DTS: dangerous to self, GAF: Global Assessment of Functioning

times more likely to be diagnosed as having schizophrenia spectrum disorder than Caucasians. For affective disorder, six variables (family psychiatric history, length of hospitalization, marital status, ethnicity, living situation, and insurance) were found to be significantly associated with the diagnosis. Affective disorder increased with presence of family psychiatric history $(\mathrm{OR}=0.655 ; 95 \% \mathrm{CI}, 0.52-0.824)$, failure of marriage $(\mathrm{OR}=1.426$; 95\% $\mathrm{CI}, 1.211-1.679)$, not-homelessness (i.e., home-staying; $\mathrm{OR}=1.817 ; 95 \% \mathrm{CI}, 1.410-2.342$ ), and quality insurance (OR=1.326; 95\% CI, 1.155-1.523). African Americans and Hispanics were 50\% less likely to be diagnosed as having an affective disorder (OR=0.56; 95\% CI, 0.4710.666).

Comparison of the logistic regression model chi-square values with and without demographic variables showed significant improvement in the prediction of diagnoses with the addition of demographic variables to clinical variables as predictors $\left(\chi^{2}\right.$ difference $=291.29-166.73=124.56, \mathrm{df}=15-5=10$, 
Table 3. Chi-square analyses of ethnic and gender differences

\begin{tabular}{|c|c|c|c|c|c|c|}
\hline \multirow{2}{*}{ Variables } & \multicolumn{2}{|c|}{ Caucasians } & \multicolumn{2}{|c|}{ Hispanics } & \multicolumn{2}{|c|}{ African Americans } \\
\hline & Males $(\mathrm{N}=609)$ & Females $(\mathrm{N}=589)$ & Males $(\mathrm{N}=299)$ & Females $(\mathrm{N}=308)$ & Males $(\mathrm{N}=123)$ & Females $(\mathrm{N}=123)$ \\
\hline \multicolumn{7}{|l|}{ Schizophrenia spectrum } \\
\hline Count (Exp. Count) & $252(279.2)$ & $198(262.1)$ & $199(141.0)$ & $136(137.2)$ & $87(53.4)$ & $69(56.1)$ \\
\hline Std. Residual & -1.7 & -4.1 & 4.7 & -0.3 & 4.5 & 1.5 \\
\hline \multicolumn{7}{|l|}{ Affective disorder } \\
\hline Count (Exp. Count) & $275(243.8)$ & $299(228.9)$ & $68(123.0)$ & $125(119.8)$ & $14(46.6)$ & $39(48.9)$ \\
\hline Std. Residual & 1.9 & 4.4 & -5.1 & 0.3 & -4.8 & -1.6 \\
\hline \multicolumn{7}{|c|}{$\chi^{2}=138.759, \mathrm{df}=5, \mathrm{p}<0.001$} \\
\hline
\end{tabular}

Table 4. Logistic regression analysis of variable predicting schizophrenia spectrum disorder and affective disorder

\begin{tabular}{|c|c|c|c|c|c|c|}
\hline \multirow{2}{*}{ Variables } & \multicolumn{3}{|c|}{ Schizophrenia spectrum disorder } & \multicolumn{3}{|c|}{ Affective disorder } \\
\hline & Wald $\chi^{2}$ & $\mathrm{p}$ & Odds ratio & Wald $\chi^{2}$ & $\mathrm{p}$ & Odds ratio \\
\hline \multicolumn{7}{|l|}{ Clinical } \\
\hline Type of admission & 2.693 & 0.101 & 0.684 & 1.307 & 0.253 & 1.281 \\
\hline Discharge GAF & 8.503 & 0.004 & 0.831 & 0.852 & 0.356 & 1.058 \\
\hline Family psychiatric history & 12.821 & $<0.001^{*}$ & 1.545 & 13.015 & $<0.001^{*}$ & 0.655 \\
\hline Length of hospitalization & 43.56 & $<0.001^{*}$ & 1.081 & 15.544 & $<0.001^{*}$ & 0.955 \\
\hline Physician race & 0.061 & 0.806 & 1.016 & 0.294 & 0.588 & 0.967 \\
\hline \multicolumn{7}{|l|}{ Demographic } \\
\hline Age & 3.808 & 0.051 & 1.262 & 1.575 & 0.209 & 1.158 \\
\hline Gender & 16.613 & $<0.001^{*}$ & 0.609 & 5.686 & 0.017 & 1.328 \\
\hline Employment & 7.946 & 0.005 & 0.729 & 7.586 & 0.006 & 1.315 \\
\hline Education & 12.919 & $<0.001^{*}$ & 0.799 & 4.816 & 0.028 & 1.139 \\
\hline Marital status & 19.302 & $<0.001^{*}$ & 0.672 & 18.047 & $<0.001^{*}$ & 1.426 \\
\hline Ethnicity & 43.891 & $<0.001^{*}$ & 1.758 & 43.320 & $<0.001^{*}$ & 0.560 \\
\hline Living situation & 10.648 & $0.001^{*}$ & 0.66 & 21.309 & $<0.001^{*}$ & 1.817 \\
\hline Insurance & 17.096 & $<0.001^{*}$ & 0.743 & 15.999 & $<0.001^{*}$ & 1.326 \\
\hline Residence & 0.002 & 0.965 & 1.005 & 1.323 & 0.250 & 0.874 \\
\hline Religion & 0.092 & 0.761 & 1.036 & 0.619 & 0.432 & 1.095 \\
\hline Model $\chi^{2}(\mathrm{df}), \mathrm{p}$-value & \multicolumn{3}{|c|}{$291.29(15), \mathrm{p}<0.001$} & \multicolumn{3}{|c|}{$240.4(15), \mathrm{p}<0.001$} \\
\hline
\end{tabular}

Aggression type is not included due to its violation of the assumption (adequacy of expected frequency). Significant results are marked by an asterisk (*). GAF: Global Assessment of Functioning

$\mathrm{p}<0.001$ for schizophrenia; $\chi^{2}$ difference $=240.4-80.7=159.7$, $\mathrm{df}=15-5=10, \mathrm{p}<0.001$ for affective disorder). It was reasonably concluded that these demographic variables had a significant effect on the prediction of the diagnosis (prediction success rate increased from $62.8 \%$ to $69.2 \%$ for schizophrenia, and from $61.2 \%$ to $67.9 \%$ for affective disorder as a result of the addition of demographic variables). That is, demographic variables significantly enhanced prediction of psychiatric diagnosis after prediction by clinical variables.

\section{DISCUSSION}

In this study of psychiatric inpatients in a large sample $(\mathrm{n}=$
2,051), we examined less chronically ill patients admitted for acute hospitalization, which consisted of both adults at their first admission and those with multiple prior psychiatric hospitalizations.

The ethnic composition of subjects was $58.4 \%$ Caucasian, 29.6\% Hispanic, and 12\% African American and 86\% (n= 1,760 ) of subjects were either a schizophrenia spectrum disorder or an affective disorder. We found Hispanics and African Americans displayed a higher rate of schizophrenia spectrum disorder and a lower rate of affective disorder compared with Caucasians, who had a higher rate of affective disorder. These results are generally consistent with previous studies. ${ }^{1-12}$ There were no finding of ethnic differences in anxiety disor- 
der, alcohol and substance use disorder, and personality disorder. Specifically, in this study, Hispanics were younger and less educated compared with Caucasians. Other investigations of the mental health system have reported that Hispanics and African Americans had lower mean incomes, less education, and were younger, less likely to be married and more likely uninsured. ${ }^{14}$

Our result showed Hispanic and African American males had far more Schizophrenia spectrum disorder than Caucasians while Caucasian females had a higher incidence affective disorder than other ethnic groups. Patient gender and ethnicity were primary predictors of receiving a clinical diagnosis of a schizophrenia spectrum disorder. Our findings replicated those of several other studies showing higher rates of schizophrenia spectrum disorder among African Americans and Hispanics. If at least some of these patients are being overdiagnosed with schizophrenia spectrum disorder, why is this result occurring?

In previous investigations, the overdiagnosis of schizophrenia was thought to be the result of misdiagnosis rather than any ethnic difference in prevalence. ${ }^{5,8-13,18,26}$ Strakowski et al. reported that information variance (differences in the availability of patient information) was the cause of diagnostic disagreement in $58 \%$ of cases and was associated with patient race. ${ }^{8}$ Criterion variance (differences in how clinical findings are interpreted) occurred in $42 \%$ of cases and was not associated with patient race. These suggest that because Schneiderian first rank or other psychotic symptoms may be common in African American patients, affective symptoms may be missed. ${ }^{8,12,20}$

When a semi-structured instrument and the Diagnostic and Statistical Manual (DSM) of the American Psychiatric Association criteria were used, Caucasians were more likely than African Americans to receive a diagnosis of bipolar disorder and less likely to be diagnosed with schizophrenia, suggesting that the difference of basic expression of symptom between African American and Caucasian patients, and clinician bias causes the diagnostic discrepancy toward schizophrenia. ${ }^{7}$ A review of records of 76 bipolar (DSM-III) patients showed that Hispanic and African American patients may be at higher risk than Caucasians for misdiagnosis as schizophrenia, particularly if they are young and experience auditory hallucinations during affective episodes. ${ }^{13}$

Another study, comparing 63 Caucasian and 53 MexicanAmerican patients of schizophrenia on ten psychiatric symptoms found that Mexican-Americans were more likely to report physical symptoms, whereas Caucasians reported greater frequency of persecutory delusions, nervous tension, and blunted affect, suggesting that presentation of schizophrenia can be influenced by sociocultural factors. ${ }^{22}$ On the other hand,
Minsky et al. reported that Hispanics were more likely to be clinically diagnosed as having major depression than African American and Caucasians, despite higher levels of self-reported psychotic symptoms. ${ }^{24}$ The findings of previous researchers showed that using a structured interview that is based on defined DSM criteria did not eliminate racial disagreements in diagnostic outcomes. ${ }^{4,7,13,27}$ Adebimpe provides that clinicians and researchers may want to consider the suggestions for compiling evidence-based DSM-V footnotes, because new guidelines may or may not capture the unique scenario in which African American patients have been systematically overdiagnosed with schizophrenia and underdiagnosed with affective disorder for more than 30 years. ${ }^{18}$

The differences in rates of diagnosis may also be due to referral biases. ${ }^{2}$ In Kern County, Ethnic minority group is African American, who make up 5.1\% of the adult population. Caucasians constitute $63.7 \%$ of adult, and Hispanic or Latino of any race account for $44.4 \%$ of adult population. ${ }^{25}$ In our study, the ethnic composition of subjects was $58.4 \%$ Caucasians, 29.6\% Hispanic, and 12\% African American. Hispanics are slightly underrepresented. Previous investigations showed that African Americans and Hispanics are underrepresented in mental health service care, compared with Caucasians, and Hispanics were more likely delayed care. ${ }^{14,24,28}$ This difference was primarily related to ethnicity and the use of Spanish as a primary language. ${ }^{28}$ Language barrier like English-Spanish translation and interpretation between Hispanic patients and non-Spanish speaking clinicians may influence inadequate diagnosis and clinical treatment. ${ }^{24,28}$ Adebimpe provided that Caucasians were diagnosed with $15.6 \%$ affective disorder, $31.5 \%$ schizophrenia, African Americans were diagnosed with $7.7 \%$ affective disorder, $32.4 \%$ schizophrenia, and Hispanics of any race were diagnosed with $15.2 \%$ affective disorder, $43.9 \%$ schizophrenia at State and County mental hospital. ${ }^{18}$ According to past psychiatric history, Hispanic and African American patients had a higher rate of schizophrenia spectrum disorder and a lower rate of affective disorder compared with Caucasian patients, who had a higher rate of affective disorder, suggesting that past psychiatric history may influence clinicians to diagnose similar disorders as previously. This could alter the diagnostic distribution of psychiatric inpatients, although our result does not account for the higher rate in schizophrenia spectrum disorder in African American and Hispanic patients.

Unlike previous studies, ${ }^{17}$ our result showed ethnic differences still existed even after controlling for the socioeconomic level. However, using insurance status as approximation of socioeconomic status does not seem to satisfactorily address the contribution of socioeconomic status to the disproportionate diagnostic pattern. We suggest that ethnic differences are influenced by cultural variance and correctable causes ra- 
ther than socioeconomic status. Correctable causes as dependent variables ${ }^{18}$ include generic disrespect, driving while black, police brutality beyond the call of duty, Census-incongruent juries, prison population disparities, death row disparities, education disparities, home ownership disparities, taxation value disparities (uncompensated: past, present, future) within causes of Census-incongruent ethnic disparities. The authors conclude that psychiatric morbidity among Mexican Americans is primarily influenced by cultural variance rather than socioecomonic status or urban versus rural residence. ${ }^{29}$ Marin and Escobar recommend to not allow cultural-specific information to obscure the individual patient, which can occur if the healthcare provider treats the information stereotypically. ${ }^{28}$

As for the race of the clinicians, our study showed that Asian and Hispanic psychiatrists tend to diagnose schizophrenia spectrum disorders more frequently than Caucasian psychiatrists, while Caucasian psychiatrists tend to diagnose affective disorder more frequently than other psychiatrists. Cultural differences between clinicians and African American patients may also lead to misdiagnosis. ${ }^{18-20}$ Another investigation of Strakowski et al. showed African males with affective psychoses appeared to be at higher risk for a misdiagnosis of schizophrenia than other patients and the rate of disagreement between senior and junior clinicians was higher for African American than Caucasian patients. ${ }^{20}$ Auditory hallucinations are more prominent in African Americans than Caucasians and are associated with depression and bipolar disorder, suggesting that risk of overdiagnosis of schizophrenia and underdiagnosis of affective disorder may be linked to misinterpretation of culturally based psychotic symptom..$^{18}$ African American psychiatrists made fewer errors of misdiagnosis as schizophrenia African American patients than Caucasian psychiatrists because of less sociocultural distance. But Hispanic patients were not protected from misdiagnosis by having a Hispanic or Spanish speaking psychiatrist because of strict adherence of DSM-III. ${ }^{6,13}$

We found that demographic variables significantly enhanced prediction of psychiatric diagnosis after prediction by clinical variables. Some specific demographic profiles (e.g., young African American males and elderly African American immigrant females) have predictive power, causes of Censusincongruent ethnic disparities. ${ }^{18}$ Our study showed low education level, failure of marriage, not-homelessness (home-staying), and low quality insurance were associated with the diagnosis of schizophrenia spectrum disorders. African American patients were three times more likely to be diagnosed as having schizophrenia spectrum disorders than Caucasians.

There is convincing evidence that social risk factors play an important role in the development of schizophrenia. Especially, males who have more stronger family history of psy- chosis tend to have an earlier age of onset compared to those cases with less genetic risk. ${ }^{30}$ Unlike previous studies, ${ }^{31,32}$ family psychiatric history patients were not significantly associated with schizophrenia spectrum disorders. This may be Hispanics and African Americans were more frequently diagnosed with schizophrenia spectrum disorders than Caucasians with family psychiatric history. The risk for schizophrenia has been found to be higher in men than women, and the incidence risk ratio was 1.3-1.4. But in developing countries, significant sex differences have not been found in incidence. ${ }^{31}$ Possible risk factors for schizophrenia in childhood and adolescence are disturbances of early development, urbanization, migration, premorbid cannabis abuse, premorbid cognitive and scholastic performance, neuroanatomical abnormalities. However, the link between childhood socioeconomic status and schizophrenia is not yet entirely resolved. ${ }^{31}$ Marriage had a protective effect for men and this result is consistent evidence for an association between social isolation and schizophrenia. ${ }^{30}$ Previous investigations have found a higher risk of homelessness in African Americans, compared with other ethnic groups, and in male gender, compared with women. Similarly, a diagnosis of schizophrenia or bipolar disorder, and poorer functioning has been associated with homelessness. ${ }^{33-35}$ Hispanics and Asian Americans were less likely to be homeless. ${ }^{34}$

According to affective disorder, presence of family psychiatric history, failure of marriage, not-homelessness (home staying), and holding quality insurance were significantly associated with affective disorders. Hispanic and African American patients were $50 \%$ less likely to be diagnosed as having affective disorder. Divorce or job loss are up to 3 times more likely to develop a major depressive disorder than those without such event. ${ }^{36}$ Family psychiatric history, being unemployed, divorce or separated, single mothers, stressful life events, and lower socioeconomic status are significant risk factor for affective disorder. ${ }^{30,37-40}$ Brown and Moran followed up single and married mothers over a 2-year period during which time rates of psychosocial risk factors, onset of depression and experience of chronic episodes, which risk of onset of depression was double among single mothers. ${ }^{37}$

There are several limitations in our study. Despite the relatively large sample size $(n=2,051)$, the findings represent clinical experiences of psychiatric inpatients in one county mental hospital without result of outpatient clinics. There were relatively modest sample sizes of Hispanics and African Americans. Our study design was retrospective. Many patients in this study were county residents, and most of patients resided in inner-city area in Kern County. This restriction limits the generalization of the results to similar county populations. In addition, in this study we did not account for use of self-re- 
ports, presenting symptoms, unstructured or structured instruments, information of the Hispanic immigrants, causal relationship between ethnicity and diagnosis. We suppose that undocumented Hispanic immigrants are less likely to be found in the patients because they are unlikely to have either public or private insurance, which limits their access. Without another source of reliable data, such as independently obtained diagnoses based on unstructured and structured clinical interview, it is not possible to confirm the accuracy of clinical diagnoses of minority groups. The possible criterion, information variance, and referral bias leading to diagnostic bias needs to be tested in future controlled empirical studies. Despite these limitations, our findings provide the various factors that contribute to ethnic differences in clinical diagnoses in psychiatric inpatients.

We conclude that our finding replicated those of several other studies showing higher rates of schizophrenia spectrum disorder among African Americans and Hispanics. This study identified different psychiatric diagnostic pattern among African Americans, Hispanics and Caucasians. In summary, schizophrenia spectrum disorder was diagnosed more frequently among African American and Hispanic males, while affective disorder was diagnosed much more frequently among Caucasian females. These associations persisted when controlled for socioeconomic status, using insurance status as an approximation of socioeconomic status. There were no ethnic differences in alcohol and substance use disorder, anxiety disorder, and personality disorder. Demographic variables significantly enhanced the prediction of psychiatric diagnosis after prediction by clinical variables.

Further investigations using unstructured or structured interviews and self-reported instruments are required to determine the accuracy and applicability of these findings, and to identify other factors that contribute to psychiatric diagnoses.

\section{REFERENCES}

1. Bell CC, Mehta H. The misdiagnosis of black patients with manic depressive Illness. J Natl Med Assoc 1980;72:141-145.

2. Delbello MP, Lopez-Larson MP, Soutullo CA, Strakowski SM. Effects of race on psychiatric diagnosis of hospitalized adolescents: a retrospective chart review. J Child Adolesc Psychopharmacol 2001;11:95-103.

3. Flaskerud JH, Hu LT. Relationship of ethnicity to psychiatric diagnosis. J Nerv Ment Dis 1992;180:296-303.

4. Keisling R. Underdiagnosis of manic-depressive illness in a hospital unit. Am J Psychiatry 1981;138:672-673.

5. Lawson WB, Helper N, Holladay J, Cuffel B. Race as a factor in inpatient and outpatient admissions and diagnosis. Hosp Community Psychiatry 1994;45:72-74.

6. Neighbors HW, Jackson JS, Campbell L, Williams D. The influence of racial factors on psychiatric diagnosis: a review and suggestions for research. Community Ment Health J 1989;25:301-311.

7. Neighbors HW, Trierweiler SJ, Ford BC, Muroff JR. Racial differences in DSM diagnosis using a semi-structured instrument: the importance of clinical judgment in the diagnosis of African Americans. J Health
Soc Behav 2003;44:237-256.

8. Strakowski SM, Hawkins JM, Keck PE Jr, McElroy SL, West SA, Bourne ML, et al. The effects of race and information variance on disagreement between psychiatric emergency service and research diagnosis in first-episode psychosis. J Clin Psychiatry 1997;58:457-463.

9. Strakowski SM, Flaum M, Amador X, Bracha HS, Pandurangi AK, Robinson OJ, et al. Racial differences in the diagnosis of psychosis. Schizophr Res 1996;21:117-124.

10. Strakowski SM, Lonczak HS, Sax KW, West SA, Crist A, Mehta R, et al. The effects of race on diagnosis and disposition from a psychiatric emergency room. J Clin Psychiatry 1995;56:101-107.

11. Strakowski SM, McElroy SL, Keck PE Jr, West SA. Racial influence on diagnosis in psychotic mania. J Affect Disord 1996;39:157-162.

12. Strakowski SM, Shelton RC, Kolbrener ML. The effects of race and comorbidity on clinical diagnosis in patients with psychosis. J Clin Psychiatry 1993;54:96-102.

13. Mukherjee S, Shukla S, Woodle J, Rosen AM, Olarte S. Misdiagnosis of schizophrenia in bipolar patients: a multiethnic comparison. Am J Psychiatry 1983;40:1571-1574.

14. Wells K, Klap R, Koike A, Sherbourne C. Ethnic disparities in unmet need for alcoholism, drug abuse, and mental health care. Am J Psychiatry 2001;158:2027-2032.

15. Adebimpe VR. Overview: white norms and psychiatric diagnosis of black patients. Am J Psychiatry 1981;138:279-285.

16. Robins LN, Helzer JE, Weissman MM, Orvarschel H, Gruenberg E, Burke JD Jr, et al. Lifetime prevalence of specific psychiatric disorders in three sites. Arch Gen Psychiatry 1984;41:949-958.

17. Robins LN, Regier DA. Psychiatric Disorders in America: the Epidemiologic Catchment Area Study. New York, NY: Free Press; 1991.

18. Adebimpe VR. A second opinion on the use of white norms in psychiatric diagnosis of black patients. Psychiatr Ann 2004;34:543-551.

19. Littlewood R. Psychiatric diagnosis and racial bias: empirical and interpretative approaches. Soc Sci Med 1992;34:141-149.

20. Strakowski SM, Keck PE Jr, Arnold LM, Collins J, Wilson RM, Fleck DE, et al. Ethnicity and diagnosis in patients with affective disorders. J Clin Psychiatry 2003;64:747-754.

21. Adebimpe VR, Chu CC, Klein HE, Lange MH. Racial and geographic differences in the psychopathology of schizophrenia. Am J Psychiatry 1982;139:888-891.

22. Weisman AG, Lopez SR, Ventura J, Nuechterlein KH, Goldstein MJ, Hwang S. A comparison of psychiatric symptoms between Anglo-Americans and Mexican-Americans with schizophrenia. Schizophr Bull 2000; 26:817-824.

23. Kennedy N, Boydell J, van Os J, Murray RM. Ethnic differences in first clinical presentation of bipolar disorder: results from an epidemiological study. J Affect Disord 2004;83:161-168.

24. Minsky S, Vega W, Miskimen T, Gara M, Escobar J. Diagnostic patterns in Latino, African American, and European American psychiatric patients. Arch Gen Psychiatry 2003;60:637-644.

25. US Census Bureau. The Hispanic Population. Census Brief; 2000.

26. Raskin A, Crook TH, Herman KD. Psychiatry history and symptom differences in black and white depressed inpatients. J Consult Clin Psychol 1975;43:73-80.

27. Strakowski SM, Adler CM, DelBello MP. Volumetric MRI studies of mood disorders: do they distinguish unipolar and bipolar disorder? Bipolar Disord 2002;4:80-88.

28. Marin H, Escobar JI. Special issues in the psychopharmacological management of Hispanic Americans. Psychopharmacol Bull 2001;35: 197-212.

29. Vega WA, Kolody B, Aguilar-Gaxiola S, Alderete E, Catalano R, Caraveo-Anduaga J. Lifetime prevalence of DSM-III-R psychiatric disorders among urban and rural Mexican Americans in California. Arch Gen Psychiatry 1998;55:771-778.

30. Sadock BJ, Sadock VA. Kaplan and Sadock's Comprehensive Textbook of Psychiatry, 10th Edition. Philadelphia: Lippincott Williams and Wil- 
kins; 2007.

31. Mäki P, Veijola J, Jones PB, Murray GK, Koponen H, Tienari P, et al. Predictors of schizophrenia--a review. Br Med Bull 2005;73-74:1-15.

32. Tienari P, Wynne LC, Läksy K, Moring J, Nieminen P, Sorri A, et al. Genetic boundaries of the schizophrenia spectrum: evidence from the Finnish Adoptive Family Study of Schizophrenia. Am J Psychiatry 2003;160: 1587-1594.

33. Caton CL, Shrout PE, Dominguez B, Eagle PF, Opler LA, Cournos F. Risk factors for homelessness among women with schizophrenia. Am J Public Health 1995;85:1153-1156.

34. Folsom DP, Hawthorne W, Lindamer L, Gilmer T, Bailey A, Golshan S, et al. Prevalence and risk factors for homelessness and utilization of mental health services among 10,340 patients with serious mental illness in a large public mental health system. Am J Psychiatry 2005;162:370376.
35. Herman DB, Susser ES, Jandorf L, Lavelle J, Bromet EJ. Homelessness among individuals with psychotic disorders hospitalized for the first time: findings from the Suffolk County Mental Health Project. Am J Psychiatry 1998;155:109-113.

36. Brown GW, Bifulco A, Harris TO. Life events, vulnerability and onset of depression: some refinements. Br J Psychiatry 1987;150:30-42.

37. Brown GW, Moran PM. Single mothers, poverty and depression. Psychol Med 1997;27:21-33.

38. Harkness KL, Luther J. Clinical risk factors for the generation of life events in major depression. J Abnorm Psychol 2001;110:564-572.

39. Kendler KS, Karkowski LM, Prescott CA. Causal relationship between stressful life events and the onset of major depression. Am J Psychiatry 1999;156:837-841.

40. Turner RJ, Wheaton B, Lloyd DA. The epidemiology of social stress. Am Sociol Rev 1995;60:104-125. 